\title{
A Resident Perspective on Adding Value as Radiologists
}

\author{
Ziga Cizman, MD, MPH ${ }^{\mathrm{a}}$, Mark Hammer, $\mathrm{MD}^{\mathrm{b}}$, Brett Mollard, $\mathrm{MD}^{\mathrm{c}}$, Rustain Morgan, $\mathrm{MD}^{\mathrm{d}}$, Zachary \\ Ballenger, $\mathrm{MD}^{\mathrm{e}}$, Gabriel J. Runner, $\mathrm{MD}^{\mathrm{f}}$, Darel E. Heitkamp, MD ${ }^{\mathrm{e}}$, Charles Resnik, $\mathrm{MD}^{\mathrm{g}}$ \\ a Department of Radiology and Imaging Sciences, Emory School of Medicine, 1364 Clifton Road \\ NE, Atlanta, GA 30322
}

b Department of Radiology, University of Pennsylvania School of Medicine, Philadelphia, USA

c Department of Radiology and Biomedical Imaging, University of California-San Francisco, San Francisco, USA

d Department of Radiology, Duke University Medical Center, Durham, USA

e Department of Radiology and Imaging Sciences, Indiana University School of Medicine, Indianapolis, USA

f Department of Radiology, University of Washington School of Medicine, Seattle, USA

g Department of Diagnostic Radiology and Nuclear Medicine, University of Maryland School of Medicine, Baltimore, USA

\begin{abstract}
During the annual 46th annual American Alliance of Academic Chief Residents in Radiology (A3CR2) meeting in New Orleans, chief residents discussed the role of residents within American College of Radiology 3.0 campaign. Our discussion was directed toward the evolving role of fourth-year radiology residents and how we might improve their training to better prepare them to add value as both leaders and radiologists. The ideas resulting from our Problem Solving session were divided into three categories:
\end{abstract}

This is the author's manuscript of the article published in final edited form as:

Cizman, Z., Hammer, M., Mollard, B., Morgan, R., Ballenger, Z., Runner, G. J., ... Resnik, C. (2016). A Resident Perspective on Adding Value as Radiologists. Academic Radiology, 23(4), 517-520.

http://doi.org/10.1016/j.acra.2015.12.006 
clinical presence in the wards and subspecialty clinics; visibility to clinicians and patients; and the education of medical students, residents, and advanced practice clinicians to aid in realizing the long-term goals of Imaging 3.0.

Key Words: Resident; education; value; radiology

\section{Introduction}

With the introduction of Imaging 3.0, the American College of Radiology has set an agenda for radiologists to seek out leadership roles and optimize high-value imaging care in our constantly changing health-care system. This journey will include both established radiologists and thousands of newly trained residents and fellows, who will become the future leaders of our profession. Residency training curricula should grow to provide training for the new roles that radiologists would need to adopt in this model. If we plan to add value to the health-care community as radiologists, then we as residents need to practice the methods by which we intend to implement these effects.

The American Alliance of Academic Chief Residents in Radiology $\left(\mathrm{A}^{3} \mathrm{CR}^{2}\right)$ is an affinity group with the Association of University Radiologists dedicated to advancing the interests of radiology residents in collaboration with other leaders within academic radiology. Shortly after inception in 1968 by Dr. Malcolm Jones, $\mathrm{A}^{3} \mathrm{CR}^{2}$ developed a series of sessions at the annual Association of University Radiologists meeting entitled "Problem Solving," designed to examine the difficult issues facing residency programs throughout the country. Chief residents select a topic for discussion at the preceding $\mathrm{A}^{3} \mathrm{CR}^{2}$ meeting, brainstorm for potential solutions, and discuss the results at a roundtable session with members of the Association of Program Directors in Radiology and the Society of Chairs of Academic Radiology Departments.

This year, our focus was directed toward the evolving role of fourth-year radiology residents and how we might improve their training to better prepare them to add value as both leaders and radiologists. The ideas resulting from our Problem Solving session can be divided into the following three categories: 
clinical presence in the wards and subspecialty clinics; visibility to clinicians and patients; and the education of medical students, residents, and advanced practice clinicians (APCs). Through reorganization of the fourth-year curriculum, we can begin to implement these ideas in the academic medical environment to ensure optimal educational and clinical outcomes.

\section{Clinical Presence in the Wards and Subspecialty Clinics}

One important requisite to establishing the value of the radiologist in the ever-changing health-care climate is face-to-face communication with our colleagues in other specialties, what is termed clinical presence. Clinical presence is important because radiologists are best suited to answer clinical imaging questions, which is done most effectively if we are able to communicate directly and meaningfully with the clinicians. Although this kind of direct communication was once more common, the advent of electronic image storage and distribution (i.e. picture archiving and communication system) has made it easy for clinicians to view radiology studies from clinical care areas, without any face-to-face interaction with a radiologist (1).

Radiologists have been tackling these challenges since at least the 1980s, with numerous papers 2 and 3 discussing the importance of radiology's participation in patient care. One strategy for improving clinical presence is the embedded reading room. These radiology spaces are located within or adjacent to patientcare areas dedicated to a specific subspecialty; for example, an embedded neuroradiology reading room located within a neurology clinic. The full impact of this strategy has not been extensively studied, but one institution found that embedded reading rooms were associated with much higher rates of face-to-face clinician-radiologist interactions and clinician-initiated interactions (4). Although the idea of embedded reading rooms may increase our clinical interactions, Rosenkrantz et al found that it could also increase our visibility to patients, with $5 \%$ of the integrated reading room consults involving direct image review by the radiologist with the patient (5). Although radiology residents themselves have no control over reading room location, they may be called upon to support this model of service delivery and will become leaders faced with making these decisions in the future. 
A natural extension of the embedded reading room concept is the embedded radiology resident. This resident would accompany clinicians during their daily clinical duties (e.g. inpatient medicine rounds) and function to review patient imaging, teach clinicians about image interpretation, and educate clinicians about study appropriateness 6 and 7. The immediate availability of the radiology resident during rounds could encourage conversations regarding imaging appropriateness and may even prevent redundant or unindicated imaging examinations from being ordered by the clinical team. While the embedded radiologist may represent an ideal way to interact with clinical teams, it is not always feasible from a residency scheduling perspective.

To mitigate this staffing constraint, some institutions have developed a radiology consultation pager that serves as a single point of contact for clinicians with questions about imaging (8). In either model, removing barriers to clinician-radiologist communication is likely to improve patient care and clinician satisfaction. Radiology residents can play an instrumental role in setting up clinical rounding or consultation services at their programs. Resident participation and enthusiasm are key ingredients for the success of these types of programs.

Additional potential roles for senior radiology residents are involvement and organization of tumor boards and multidisciplinary conferences under the guidance of faculty. These conferences represent another important venue for radiologists and clinicians to foster collaborative relationships and learn clinical management expertise. This experience will also serve as template on how to become involved in these conferences after graduation.

Although fears of decreasing reimbursement rates and a saturated job market may be at least partially responsible for the decline in medical student interest, lack of sufficient medical student exposure to radiology may also represent an important contributing factor. Medical students frequently have limited radiology exposure during the nonclinical years of medical school, with only gradual glimpses of radiology through the eyes of other specialties (9). Medical students typically do not have the opportunity to rotate through radiology until their clerkship years of medical school, limiting exposure to the field 
before residency application deadlines in the fall of their fourth year. Increasing the clinical presence of radiologists may also increase and strengthen interactions with medical students, which is important given the $28 \%$ decline in radiology residency applications over the last 5 years and the substantial increase in the number of unmatched radiology residency positions over the last 2 years (84 in 2014 and 137 in 2015) 10, 11 and 12.

\section{Visibility to Clinicians and Patients}

Radiologists primarily work in reading rooms rather than in general patient-care areas due to increasing case volume and the fee-for-service model, where efficiency is an important driving factor. However, this paradigm has come at a cost: $50 \%$ of Americans are now uncertain whether a radiologist interprets or acquires imaging studies (13). One study found that only $14 \%$ of patients knew that radiologists were physicians; most believed that radiologists simply have a degree in radiography (13). It is clear that we, as a field, need creative ways to improve visibility with regard to both our colleagues and our patients.

Improving the visibility of radiologists requires a multipronged approach. Social media and other Webbased venues have become increasingly important tools to increase the brand of institutions, companies, and causes in the 21 st century. Residents are typically more comfortable with social media and may be best equipped to help radiology departments embrace the role of social media in health care. There are multiple benefits to having a social media presence, including education, communication, and collaboration with patients and referring providers. In addition, online resources could include providing services via telecommunication to underserved areas, Web sites for preprocedural information, and Twitter to update radiology wait times, or traffic and parking alerts. Additional ideas for increasing patient awareness of radiologists include improving departmental signage via use of monitors or tablets to introduce the radiology staff to patients and clinicians, educating patients about available services, and providing up-to-date wait times and study information while patients are in the radiology waiting room. 
The availability of radiology reports to patients via health information portals offers a new opportunity for radiologists to engage patients. These opportunities can be leveraged into actual clinic appointments with radiologists for patients who wish to review their reports and images in person with a radiologist. This approach has allowed mammography to flourish and breast imagers to form bonds with their patients. Unfortunately, many other radiologists have not seized this opportunity to get involved despite evidence suggesting patients desire our involvement 14, 15 and 16. Several institutions, such as the Massachusetts General Hospital, have started radiology consultation clinics where radiologists and/or trainees discuss imaging findings with patients as well as more general issues such as the effects of radiation exposure and alternative imaging modalities (16). Such clinics have received positive feedback from patients.

Radiology reports are the most visible product a radiologist produces, and therefore a radiologist's value is currently dependent on report quality. As clinical subspecialization has increased, some nonradiology specialists may attempt to interpret their own studies (5). One idea to improve our value is to create tailored radiologic reports based on a referring provider's clinical question or specified disease process. For example, current literature suggests primary care provides are less likely to read an entire report, which may necessitate pertinent incidental discoveries being listed in the impression (17). Through open communication and utilization of embedded reading rooms and consult services, we can use clinician feedback to optimize our reports and provide greater clinical relevancy.

Finally, radiologists should take more active roles in hospital and university committees. Radiology residents can participate in graduate medical education-related committees as well as committees focusing on patient safety, communication, and technologic infrastructure. In fact, resident involvement in patient safety committees is an important part of the Accreditation Council for Graduate Medical Education next accreditation system (Clinical Learning Environment Review) (18). By participating in hospital-wide committees, radiologists help shape policy, meet other health-care members, and improve their visibility within the hospital. This important activity promotes collaboration and fosters new relationships with other health-care professionals. 


\section{Education of Medical Students, Residents, and APCs}

Radiology education represents an opportunity for residents to add substantial value to the field of medicine. As residents, we can organize in an effort to educate medical students, residents both within and outside of radiology, patients, and APCs. Radiology residents and faculty have astute knowledge of anatomy and are well versed in pathophysiology. As such, it is imperative that radiologists participate in the preclinical medical school curricula, teaching clinically relevant anatomy and pathophysiology that medical students will use in their future practice. This curriculum change would demonstrate radiology's critical role in medicine early in students' medical education (19).

As an unintended consequence of the digital revolution, radiologists have transitioned from integral members of the medical team to a commodity. During this transition, interdisciplinary relationships have suffered. Surgery residents are largely taught radiology by surgeons. Internal medicine residents learn how to read chest radiographs and CTs from pulmonologists. As the experts at image interpretation in the hospital, radiologists need to have a role in the education of our nonradiology colleagues. This could be accomplished by helping develop interdisciplinary conferences, which would grant us the opportunity to foster relationships with referring physicians while contributing to patient care. Participation in such conferences will permit radiologists to better understand the information necessary for referring providers to formulate treatment plans.

The increasing role of APCs in health care provides an additional avenue for engaging referring clinicians. As many health-care organizations change their practice models in response to the Affordable Care Act, the role of APCs will likely continue to expand. Moreover, a recent study by Hughes, et al found that APCs order more imaging studies as compared to primary care physicians following a routine office visit (20). To better serve our patients and add value to health care, radiologists could help integrate the American College of Radiology appropriateness criteria into a curriculum for APCs and become involved in their education. 


\section{Discussion}

We have advocated a number of proposals to help increase radiologists' value in the future of medical care. Unfortunately, implementing the changes described above will require substantial investment and commitment from the field of radiology. These changes will have an even greater impact on smaller programs where service coverage may already be spread thin. Additionally, integrating radiology education into medical school curricula and opening radiology consultation clinics may also negatively impact revenue generation for a department, as it pulls radiologists away from reading studies.

Utilizing senior radiology residents to educate referring providers, medical students, and patients seems an appropriate solution to implement many of these programs. Following the completion of the ABR Core exam in June of the third year of radiology residency, fourth-year radiology residents now have a varying amount of elective time as well as the clinical knowledge necessary to take on the role of educator. A potential radical solution would involve a change to the structure of the radiology internship requirement. A similar shift had previously been championed by the 2005 Intersociety Conference report (21). However, instead of a longer fellowship, we suggest converting the intern year into a clinical radiology year to be completed after the core exam. Thus, the new format for radiology residency would be 5 years of radiology plus a fellowship year.

A year of clinical radiology would allow senior residents to act as expert imaging consultants to their clinical colleagues, giving live interpretations and modality/protocol advice. This would expose radiology residents to the implications that their interpretations have on patient care. Integrating radiology in this way could also aid in recruiting top medical students, improving interdisciplinary relationships, and enhancing the visibility of the radiologist to the patient population. By redesigning the structure of radiology residency, we may be able to drastically alter the future of our profession.

At first glance, the role of trainees in implementing these sweeping changes appears limited; however, trainees have an important perspective and must act to spur and shape these changes to ensure the future 
success of our specialty. Although our discussion here is not meant to be all encompassing, it provides ideas for quality improvement projects that could set up a framework to discover which changes best demonstrate the value of radiology to patients, referring providers, and hospital administrators.

\section{Conclusion}

Radiology residents can aid in realizing the long-term goals of Imaging 3.0 by embracing opportunities to increase radiologist visibility to the medical community and general patient population, expanding our clinical presence as educators and consultants. Restructuring of radiology residency by delaying the clinical year to follow the core exam is a potential solution to increase radiologist visibility to the healthcare community and general patient population, while also expanding our clinical presence as educators and consultants.

\section{Acknowledgements}

2014-2015 American Alliance of Academic Chief Residents In Radiology Steering Committee, Gautham P. Reddy, Laurie May. 


\section{References}

1. Reiner B, Siegel E, Protopapas Z, et al. Impact of filmless radiology on frequency of clinician consultations with radiologists. AJR Am J Roentgenol 1999; 173:1169-1172.

2. Baker SR. The operation of a radiology consultation service in an acute care hospital. JAMA 1982; 248:2152-2154.

3. Seltzer SE, Beard JO, Adams DF. Radiologist as consultant: direct contact between referring clinician and radiologist before CT examination. AJR Am J Roentgenol 1985; 144:661-664.

4. Tillack AA, Borgstede JP. An evaluation of the impact of clinically embedded reading rooms on radiologist-referring clinician communication. J Am Coll Radiol 2013; 10:368-372.

5. Rosenkrantz AB, Lepor H, Taneja SS, et al. Adoption of an integrated radiology reading room within a urologic oncology clinic: initial experience in facilitating clinician consultations. J Am Coll Radiol 2014; $11: 496-500$.

6. Mamlouk MD, Anavim A, Goodwin SC. Radiology residents rounding with the clinical teams: a pilot study to improve the radiologist's visibility as a consultant. J Am Coll Radiol 2014; 11:326-328.

7. Baker SR, Rosenberg ZS, Adel H. The operation of a ward-based radiology consultation service. Radiology 1984; 152:331-333.

8. Levin DC, Rao VM. The declining radiology job market: how should radiologists respond? J Am Coll Radiol 2013; 10:231-233. 
9. Kapoor N, Smith SE. Association between medical school radiology curricula and application rates to US radiology residency programs. J Am Coll Radiol 2014; 11:1064-1068.

10. National Resident Matching Program. Results and data: 2015 main residency match.2015. Available at: http://www.nrmp.org/wp-content/uploads/2015/05/Main-Match-Results-and-Data-2015_final.pdf. Accessed August 8, 2015.

11. National Resident Matching Program. Results and data: 2014 main residency match. Available at: http://www.nrmp.org/wp-content/uploads/2014/04/Main-Match-Results-and-Data-2014.pdf. Accessed August 8, 2015.

12. Chen JY, Heller MT. 2014 Residency match update and call to action. J Am Coll Radiol 2014; 11:835.

13. O’Mahony N, McCarthy E, McDermott R, et al. Who's the doctor? Patients' perceptions of the role of the breast radiologist: a lesson for all radiologists. Br J Radiol 2012; 85:e1184-e1189.

14. Pahade J, Couto C, Davis RB, et al. Reviewing imaging examination results with a radiologist immediately after study completion: patient preferences and assessment of feasibility in an academic department. AJR Am J Roentgenol 2012; 199:844-851.

15. Miller P, Gunderman R, Lightburn J, et al. Enhancing patients' experiences in radiology: through patient-radiologist interaction. Acad Radiol 2013; 20:778-781. 
16. Mangano MD, Bennett SE, Gunn AJ, et al. Creating a patient-centered radiology practice through the establishment of a diagnostic radiology consultation clinic. AJR Am J Roentgenol 2015; 205:95-99.

17. Gunderman R, Ambrosius WT, Cohen M. Radiology reporting in an academic children's hospital: what referring physicians think. Pediatr Radiol 2000; 30:307-314.

18. Weiss KB, Wagner R, Bagian JP, et al. Advances in the ACGME Clinical Learning Environment Review (CLER) program. J Grad Med Educ 2013; 5:718-721.

19. Branstetter BF, Faix LE, Humphrey AL, et al. Preclinical medical student training in radiology: the effect of early exposure. AJR Am J Roentgenol 2007; 188:W9-W14.

20. Hughes DR, Jiang M, Duszak R, Jr. A comparison of diagnostic imaging ordering patterns between advanced practice clinicians and primary care physicians following office-based evaluation and management visits. JAMA Intern Med 2015; 175:101-107.

21. Dunnick NR, Applegate K, Arenson R, et al. Training for the future of radiology: a report of the 2005 Intersociety Conference. J Am Coll Radiol 2006; 3:319-324. 